\title{
The pectoralis major musculocutaneous flap in head and neck cancer reconstruction
}

\author{
José Luis Ortiz Bogado ${ }^{1}$, Felipe Guilherme da Silva Souza ${ }^{1}$, and Rogério Aparecido Dedivitis ${ }^{2 *}$ \\ ${ }^{1}$ Resident of Head and Neck Surgery at Hospital Ana Costa, São Paulo, Brazil \\ ${ }^{2}$ Department of Head and Neck Surgery, Hospital das Clínicas, University of São Paulo School of Medicine, São Paulo, Brazil \& Chairman, Departments of Head \\ and Neck Surgery, Hospital Ana Costa, Santos and Irmandade da Santa Casa da Misericórdia, Santos, Brazil
}

\begin{abstract}
Introduction: The pectoralis major flap was initially described by Ariyan in the 1970s. It has high acceptance due to its versatility for head and neck cancer reconstruction.

Methods: This flap was used for the reconstruction of 46 patients with head and neck cancer, as following: mouth/tongue reconstruction (24); pharyngoesophageal transit reconstruction (11); repair of cervical skin loss (4); pharynx reconstruction (2); repair of facial skin loss (3); repair of pharyngocutaneous fistula (1) and salvage reconstruction after rotational flap necrosis (1).
\end{abstract}

Results: Total necrosis of the flap was observed in two patients, partial necrosis in six and partial dehiscence in eleven.

Conclusions: The pectoralis major flap is functionally adequate for the repair of large surgical defects.

\section{Introduction}

The treatment of head and neck cancer often requires extensive resections and flaps for its reconstruction. Since it provides good cosmetic results, immediate reconstruction is preferable. If the use of a flap is required, its choice must be made taking into account the anatomical and functional characteristics of the tissue removed, the characteristics of the recipient and the donor site, the patient's general conditions and the experience of the surgeon [1].

The pectoralis major musculocutaneus flap (PMMF) is versatile and widely used in the head and neck region since the studies of Ariyan $[2,3]$. The pectoralis major muscle is irrigated mainly by the thoracoacromial artery with additional circulation provided by the lateral thoracic artery.

The objective of this study is to present the local complications of the use of the pectoralis major muscle flap, in large head and neck reconstructions, as well as to present the approach taken against the complications found.

\section{Methods}

We performed reconstruction with PMMF in 46 patients surgically treated during the period from January, 1995 to December, 2016 in the Department of Head and Neck Surgery of the Ana Costa Hospital, Santos and of Irmandade da Santa Casa da Misericórdia de Santos. Forty two patients were men, with the age varying from 38 to 75 , with the median of 59 years old and an average age of 60.3 .

The indications for this flap rotation were: floor of the mouth and tongue reconstruction (24 patients); reconstruction of the pharyngoesophageal transit after total pharyngolaryngectomy (11); loss of soft parts of cervical area infiltrated by squamous cell carcinoma lymph node metastasis with extra capsular spread (4); loss of soft parts of the face after tumoral resection (3); pharyngeal reconstruction (2); pharyngocutaneous fistula closure post pharyngolaryngectomy (1) and salvage reconstruction after rotational flap necrosis for mandibular reconstruction (1) - Table 1 . Five patients were previously irradiated. Most reconstructions were accomplished in the same act of the tumor surgical resection (43). The donor site could be primarily closed in most of the cases; just one case needed a skin graft.

\section{Results}

Of a total of 46 patients, $22(47.8 \%)$ presented complications after reconstruction with PMMF. The most common complication after PMMF reconstruction observed was partial loss of the skin of the flap with partial dehiscence at the suture line (11 patients) - Table 2. Four patient developed total dehiscence, 3 with fistula and 1 with exposure of reconstruction plate. No one required a second flap. Two patients required pharyngostostomy because of persistent fistula. We had 2 cases of total necrosis of the flap and six with partial necrosis. Seven patients required surgical debridement and one underwent correction of the defect with deltopectoral flap with good results. Two patients presented complications in the donor area and one presented a seroma and the other graft necrosis. A case of pharyngoesophageal transit reconstruction presented pharyngeal stenosis and required

Correspondence to: Rogério Aparecido Dedivitis, Av. Conselheiro Nébias, 444 16o andar, 11045-000 Santos, SP, Brasil, E-mail: dedivitis.hns@uol.com.br

Key words: surgical flaps, reconstructive surgical procedures, head and neck neoplasms, postoperative complications

Received: January 10, 2017; Accepted: January 28, 2017; Published: January 31, 2017 
Table 1.Indications for the pectoralis major flap rotation.

\begin{tabular}{|c|c|}
\hline Indications for the flap & Number of patients \\
\hline Tongue and floor of the mouth repair & 24 \\
\hline Pharyngoesophageal transit reconstruction & 11 \\
\hline Repair of cervical loss of skin & 4 \\
\hline Repair of facial loss of skin & 3 \\
\hline Pharynx reconstruction & 2 \\
\hline Repair of pharyngocutaneous fistula & 1 \\
\hline Salvage reconstruction after rotational flap necrosis & 1 \\
\hline Total & 46 \\
\hline
\end{tabular}

Table 2.Complications in our patients.

\begin{tabular}{|c|c|}
\hline Complications in 17 flaps & Number of patients \\
\hline Loss of skin/partial dehiscence & 11 \\
\hline Total necrosis & 2 \\
\hline Partial necrosis & 6 \\
\hline Donor site complications & 2 \\
\hline Stenosis & 1 \\
\hline Total & 22 \\
\hline
\end{tabular}

gastrostomy. Twelve patients with complications needed only conservative treatments.

\section{Discussion}

The PMMF is versatile and the reconstruction of major defects in the head and neck can be easily performed using a single time procedure. The use of a flap with non-irradiated muscle at a nonirradiated site facilitates good results.

The flap is well supported in the muscle segment in the reconstruction of intraoral defects, even in the presence of skin loss and partial dehiscence. In these cases of epidermolysis, patients tend to evolve satisfactorily with local therapy, forming granulation tissue in the area. Despite subcutaneous irrigation by arterial branches, saliva contributes to loss of the superficial layer of the skin. On the other hand, it is possible to achieve good results when using a fasciomuscular flap, that is, without using the skin of the thorax, for the repair of intraoral defects. An additional advantage of non-rotation of the chest skin along with the flap is to prevent the growth of hairs inside the mouth. However, since a good part of these patients will undergo adjuvant radiotherapy, such a therapeutic modality ends up causing depilation.

Limitations of the PMMF include restricted reach, neck contracture caused by fibrosis of the proximal muscle and frequently an unsightly bulge in the neck. Despite these drawbacks, the pectoralis major musculocutaneous flap is still commonly used in patients who are poor free flap candidates, as an additional flap in conjunction with a free flap to reconstruct massive defects, or as a secondary option in the event of a free flap failure [4].

The incidence of complications ranges from 16 to $41 \%$ [5]. In a series that evaluated 96 cases, Wadwongtham et al. [6] described greater complications of PMMC in $17.7 \%$ of the patients, including total loss of flap, partial loss of skin, orocutaneous fistula, dehiscence and plate exposure. The overall complication rate was $54.2 \%$, with the majority being conservatively treated. In a period of 20 years, Milenovic et al. [7] used 506 PMMF in 500 patients for intraoral (387 cases - 77\%) and pharyngeal reconstruction (78 cases - 15\%). Bone defects occurred in 65 patients. In $31(6 \%)$ the PMMF was used in combination with other flaps (deltopectoral, tongue, trapezius and microvascular flaps). Complications occurred in 168 flaps (33\%), however, total necrosis was seen only in 10 (2\%). Surgical treatment of complications was indicated in 87 cases $(17 \%)$. In another study of 27 patients with reconstruction for oral cavity cancer with titanium reconstruction plate and PMMF, Salvatori et al. [8] did not find a perioperative death and reported six cases of plaque exposure, being early in four cases and late in two. Two cases were surgically corrected, with a final success rate of $85 \%$. Most patients considered the aesthetic result acceptable. In a study with 25 patients, indications for PMMF by El-Marakby [9] were: oral cavity (10 patients); oropharynx and hypopharynx (5 patients) and neck or face (10 patients). Of 26 procedures, 22 were primary reconstructions and four were salvage approaches. Dehiscence, infection, hematoma, seroma, partial or total loss, fistula and complications at the donor site were verified in 15 patients $(60 \%)$, associated with indications for salvage surgery, comorbidities and indications for oral cavity. According to Chaturvedi et al. [10], in defects that require extensive reconstruction of the skin and mucosa, PMMF can be used in two layers or even in combination with the deltopectoral flap, especially when there is no condition to use a microvascular flap. In our series, we found $47.8 \%$ (22/46) of complications. The rotation of a second flap was required in 1 case and a deltopectoral flap was developed.

Reconstruction with PMMF can cause some healing delay for frequent necrosis of the most distal edge of the skin paddle; this usually doesn't require further interventions, but it sometimes increases hospital stay and costs [11]. We found 8 cases of necrosis in our series, of which seven needed surgical treatment. One of the explanations for the necrosis of the flap is the extrinsic compression of the vascular pedicle, mainly in the subcutaneous tunnel through which it passes, at the level of the clavicle.

Complications at the donor site, such as minimal dehiscence, hematoma, seroma and infection, have been infrequently observed [12]. In our series, there was a case of seroma, resolved conservatively and a case of dehiscence due to free skin graft necrosis, used to close the donor site.

The development of a pharyngocutaneous fistula remains the most common and challenging complication following total laryngectomies. It leads to delays of the adjuvant treatments and prolonged hospitalization. In the recent years, the treatment of laryngeal cancers has moved away from primary laryngectomy towards organ preservation, with the use of initial chemoradiation followed by salvage laryngectomy for subsequent disease recurrence. It is widely accepted that salvage total laryngectomies performed after radiation failure are more prone to complications as compared to laryngectomies performed upfront. Some authors have advocated for flap reconstruction at the time of laryngectomy in an effort to reinforce the primary pharyngeal suture line with vascularized tissue to prevent fistula formation. The PMMF has been the workhorse flap for this indication. While some studies show a clear reduction in pharyngocutaneous fistula rates, others suggest that the fistulae that occur are smaller and rarely need repair [13-16].

In recent years, the antebrachial free flap has replaced PMMF in the reconstruction of soft parts of the mouth and oropharynx. In studies matching two groups of patients, the lower cost of hospital stay was comparable to the higher cost of the surgical procedure of the microvascularized antebrachial flap. Thus, with comparable final costs and better functional outcome, the antebrachial flap is considered as the first option for reconstruction [17]. In spite of this, when the regional or general conditions of the patient do not permit the use of microvascular flaps, even in salvage reconstructions after dehiscence or previous failure, pedicled flaps should be considered [18]. Despite the 
widespread use of microvascular flaps, PMMF is still considered the main reconstructive option for head and neck cancer in many centers.

In patients submitted to total glossectomy with or without total laryngectomy, when comparing the anterolateral thigh flap with other flap types, no significant difference in functional outcomes was noted. However, when comparing the use of the pectoralis flap vs. all free tissue transfer, the pectoralis flap was associated with poorer outcomes in speech intelligibility and the ability to phonate. These results can be explained by the finding that pectoralis flaps were used more often when concurrent total laryngectomy was performed. No difference was noted in swallowing when comparing pectoralis flaps with free tissue reconstructions [19].

Despite the progressively more popular use of microvascular free flaps, PMMF remains a very reliable and easily option performed by the specialist. The radial antebrachial flap is probably preferable in the repair of areas that require more delicate options, such as in the reconstruction of defects of the total thickness of the buccal region. On the other hand, it is obviously difficult to work with a thick flap of obese patients and female patients with bulky breasts. Such difficulties can be overcome with the use of the fasciomuscular flap, whose thickness is smaller. In the case of female patients, the microvascular flaps avoid bad esthetic results in the breasts due to the inframammary incision of the PMMF.

Although advances in microsurgery have vastly increased the reconstructive surgeon's armamentarium for oral reconstruction, there is still a role for pedicled flaps. The PMMF remains a viable option for reconstruction of floor of mouth and tongue defects. It can be especially useful in patients with significant medical risks or lacking ideal donor free flaps [20].

In addition, the pectoral flap and the deltopectoral flaps provide the most desirable color-texture match to the head and neck region, especially in the reconstruction of the face and neck region, making them cosmetically very useful flaps [21].

\section{Conclusions}

The pectoralis major flap is functionally adequate for the repair of large surgical defects.

\section{References}

1. Mura F, Bertino G, Occhinni A (2012) Advanced carcinoma of the hypopharynx: functional results after circumferential pharyngolaryngectomy with flap reconstruction. Acta Otorhinolaryngol Ital 32: 154-157.

2. Ariyan S (1979) The pectoralis major myocutaneous flap. A versatile flap for reconstruction in the head and neck. Plast Reconstr Surg 63: 73-81. [Crossref]

3. Ariyan S, Cuono CB (1980) The use of the pectoralis major musculocutaneous flap for reconstruction of the large cervical, facial, or cranial defects. Am J Surg 140: 503.

4. Hanasono MM, Matros E, Disa JJ (2014) Important aspects of head and neck reconstruction. Plast Reconstr Surg 134: 968e-80e. [Crossref]
5. Lee YT (1980) Carotid artery protection by pectoralis major muscle flap. Am J Surg 140: 464. [Crossref]

6. Wadwongtham W, Isipradit P, Supanakorn S (2004) The pectoralis major myocutaneous flap: applications and complications in head and neck reconstruction. J Med Assoc Thai 87 Suppl 2: S95-99. [Crossref]

7. Milenovic A, Virag M, Uglesic V, Aljinovic-Ratkovic N (2006) The pectoralis major flap in head and neck reconstruction: first 500 patients. J Craniomaxillofac Surg 34: 340-343. [Crossref]

8. Salvatori P, Motto E, Paradisi S, Zani A, Podrecca S, et al. (2007) Oromandibular reconstruction using titanium plate and pectoralis major musculocutaneous flap. Acta Otorhinolaryngol Ital 27: 227-232.

9. El-Marakby HH (2006) The reliability of pectoralis major myocutaneous flap in head and neck reconstruction. J Egypt Natl Canc Inst 18: 41-50. [Crossref]

10. Chaturvedi P, Pai PS, Pathak KA, Chaukar DA, Deshpande MS, et al. (2005) Simultaneous reconstruction of large skin and mucosal defect following head and neck surgery with a single skin paddle pectoralis major myocutaneous flap. J Laryngol Otol 119: 303-305. [Crossref]

11. Deganello A, Gitti G, Parrinello G, Muratori E, Larotonda G, et al. (2013) Cost analysis in oral cavity and oropharyngeal reconstructions with microvascular and pedicled flaps. Acta Otorhinolaryngol Ital 33: 380-387. [Crossref]

12. Dedivitis RA, Guimarães AV (2002) Pectoralis major musculocutaneous flap in head and neck cancer reconstruction. World J Surg 26: 67-71. [Crossref]

13. Jing S, O'Neill T, Clibbon J. A comparison between free gracilis muscle flap and pedicled pectoralis major flap reconstructions following salvage laryngectomy. J Plast Reconstr Aesth Surg. 2014;67(1):17-22.

14. Gendreau-Lefèvre AK, Audet N, Maltais S, Thuot F (2015) Prophylactic pectoralis major muscle flap in prevention of pharyngocutaneous fistula in total laryngectomy after radiotherapy. Head Neck 37: 1233-1238. [Crossref]

15. Guimarães A, Aires F, Dedivitis R, Kulcsar M, Ramos D, et al. (2015) Efficacy of pectoralis major muscle flap for pharyngocutaneous fistula prevention in salvage total laryngectomy: A systematic review. Head Neck 38: E2317-E2321.

16. Paleri V, Drinnan M, van den Brekel MW, Hinni ML, Bradley PJ, et al. (2014) Vascularized tissue to reduce fistula following salvage total laryngectomy: a systematic review. Laryngoscope 124: 1848-1853. [Crossref]

17. de Bree R, Reith R, Quak JJ, Uyl-de Groot CA, van Agthoven M, et al. (2007) Free radial forearm flap versus pectoralis major musculocutaneous flap reconstruction of oral and oropharyngeal defects: a cost analysis. Clin Otolaryngol 32: 275-282.

18. Okazaki M, Asato H, Takushima A, Sarukawa S, Nakatsuka T, et al. (2007) Analysis of salvage treatments following the failure of free flap transfer caused by vascular thrombosis in reconstruction for head and neck cancer. Plast Reconstr Surg 119: 12231232. [Crossref]

19. Lin DT, Yarlagadda BB, Sethi RK, Feng AL, Shnayder Y, et al. (2015) Long-term Functional Outcomes of Total Glossectomy With or Without Total Laryngectomy. JAMA Otolaryngol Head Neck Surg 141: 797-803. [Crossref]

20. Rihani J, Lee MR, Lee T, Ducic Y (2013) Flap selection and functional outcomes in total glossectomy with laryngeal preservation. Otolaryngol Head Neck Surg 149: 547553. [Crossref]

21. Nishi Y, Rikimaru H, Kiyokawa K, Watanabe K, Koga N, et al. (2013) Development of the pectoral perforator flap and the deltopectoral perforator flap pedicled with the pectoralis major muscle flap. Ann Plast Surg 71: 365-371. [Crossref]

Copyright: (C2017 Dedivitis RA. This is an open-access article distributed under the terms of the Creative Commons Attribution License, which permits unrestricted use, distribution, and reproduction in any medium, provided the original author and source are credited. 